\title{
Comparación de Ecuaciones Antropométricas para Evaluar la Masa Muscular en Jugadores de Badminton
}

\author{
Comparison of Anthropometric Equations for Estimation Muscle Mass in Badminton Player
}

\begin{abstract}
"Francisco José Berral de la Rosa; **Elizabeth C. Rodríguez-Bies; ****arlos Javier Berral de la Rosa;
\end{abstract}
${ }^{* *}$ Daniel Rojano Ortega \& ${ }^{* * * *}$ Eleazar Lara Padilla

BERRAL DE LA ROSA, F. J.; RODRÍGUEZ-BIES, E. C.; BERRAL DE LA ROSA, C. J.; ROJANO ORTEGA, D. \& LARA PADILLA, E. Comparación de ecuaciones antropométricas para evaluar la masa muscular en jugadores de bádminton. Int. J. Morphol., 28(3):803-810, 2010.

RESUMEN: Actualmente la cuantificación de la masa muscular es un área de estudio que ocupa distintas disciplinas científicas tanto de la salud como de las ciencias del deporte por ser un componente de la masa corporal que está en íntima relación con aspectos de la salud y del rendimiento deportivo. Existe una gran variedad de métodos y ecuaciones para su estimación pero la aplicación de las mismas no siempre resulta la más adecuada. El propósito de nuestro estudio es determinar la masa muscular en 37 jugadores de bádminton españoles, a través de técnicas antropométricas y hallar la fórmula más apropiada para dicha población. Fueron evaluadas 55 variables antropométricas: peso, estatura. perímetros, diámetros, longitudes, alturas y pliegues cutáneos. La composición corporal estimada de los jugadores de bádminton fue de $44 \%$ para la masa muscular y de $13 \%$ para la masa grasa. Los resultados indican que la táctica de fraccionamiento tetracompartimental que mejor se ajusta y concuerda con el peso real estimado con balanza es la siguiente: Masa Residual (Wurch), Masa Ósea (Rocha), Masa Grasa (Faulkner) y Masa Muscular (Drinkwater o Kerr).

PALABRAS CLAVE: Bádminton, antropometría, composición corporal, masa muscular.

\section{INTRODUCCIÓN}

La cuantificación de la masa muscular (MM) en vivo ha cobrando un interés creciente en varias disciplinas tanto de la salud humana como en las ciencias del deporte. La necesidad de una estimación precisa se pone de manifiesto por su relación con aspectos de la salud tales como: el estado de las reservas proteicas, la capacidad termorreguladora, la inmunocompetencia y la independencia funcional, entre otros. Hoy se reconoce que la pérdida de tejido muscular asociado con el envejecimiento (sarcopenia) o enfermedades catabólicas (cáncer, VIH/SIDA, insuficiencia renal crónica, insuficiencia cardiaca congestiva, etc) está relacionada con la disminución de la calidad y la expectativa de vida. Por otro lado, en ciencias deportivas la adecuada cuantificación de la MM nos permitirá interpretar mejor los efectos anabólicos del entrenamiento físico en el ejercicio, su relación con el coste metabólico, la producción de fuerza, la capacidad de trabajo físico y el rendimiento muscular.

En las últimas décadas se han desarrollado diversas técnicas que se basan en las propiedades del músculo incluyendo el contenido de creatina (Poortmans et al., 2005), la concentración de potasio (Wang et al., 2007), ultrasonido (Sanada et al., 2006) y la conductividad eléctrica (Tanimoto et al., 2008). No obstante la mayoría de estos métodos se basan en una serie de supuestos que son difíciles de validar en vivo. Una alternativa a estos, como norma de referencia, son las técnicas de imagen como la resonancia magnética (Shen et al., 2004) y la tomografía computarizada (Fernández-Vieitez et al., 2000). Sin embargo son métodos costosos y de acceso limitado.

Profesor Titular y Director del Doctorado en Alto Rendimiento Deportivo. Director del Departamento de Deporte e Informática. Universidad Pablo de Olavide (UPO). Sevilla, España.

** Doctorando en Alto Rendimiento Deportivo. Departamento de Deporte e Informática. Universidad Pablo de Olavide (UPO). Sevilla, España.

*** Especialista en Ciencias Morfofuncionales del Deporte. Servicio Andaluz de Salud (SAS), España.

***** Profesor Titular y Director del Área de Postgrado del Instituto Politécnico Nacional de México (IPN), México DF. 
Otra posibilidad para realizar la medición total y regional del cuerpo es la absorciometría dual fotónica de rayos X (DEXA). Con estos instrumentos la exposición a la radiación es mínima. Los sistemas DEXA proporcionan una medida apendicular magra de tejidos blandos con un compartimiento libre de grasa que incluye el componente mineral óseo, el músculo y otros componentes tales como la piel, tendones y tejido conectivo (Kim et al., 2006).

De todos los métodos conocidos actualmente el antropométrico tiene una amplia difusión y aplicación por ser poco costoso y estar en constante validación, de hecho, gran parte de las ecuaciones utilizadas para la estimación de la MM están validadas en estudios de cadáveres.

El propósito de nuestro estudio es determinar mediante evidencia empírica qué fórmula es la más adecuada para estimar el componente muscular en un grupo de jugadores de bádminton y al mismo tiempo proponer una combinación de ecuaciones basándonos en la táctica de Matiegka (Cattrysse et al., 2002), con el objetivo de conseguir un fraccionamiento corporal de cuatro componentes y estimar de esta forma el peso corporal de la muestra estudiada lo más aproximado posible al obtenido mediante el uso de la balanza.

\section{MATERIAL Y MÉTODO}

El proyecto se llevó a cabo durante el X Campeonato de España de Bádminton Júnior, evaluando treinta siete jugadores de bádminton de sexo masculino. Para la selección de medidas antropométricas, técnica e instrumental de medición se tuvieron en cuenta las recomendaciones de la Sociedad Internacional para el Avance de la Cineantropometría (ISAK) en su manual, International standards for anthropometric assessment (Marfell-Jones et al., 2006).

Al estimar el componente muscular fue necesario contar con la medida de la estatura, valores de perímetros musculares y pliegues cutáneos. Aplicamos seis ecuaciones de predicción de la MM existentes en la literatura científica (Poortmans et al.; Heymsfield et al., 1982; Drinkwater, 1984; Martin et al., 1990; Kerr, 1988, Doupe et al., 1997), que posteriormente serán combinadas siguiendo la táctica de Matiegka en un modelo fraccional de cuatro componentes, masa residual (MR), masa ósea (MO), masa grasa (MG) y masa muscular (MM).

La ecuación de Lee et al. (2000) no pudo ser tenida en cuenta porque la variable para el perímetro del muslo no fue registrada como describe el autor.
Tras la estimación de la MM, aplicamos la táctica de Matiekga de fraccionamiento de la composición corporal en cuatro componentes utilizando las ecuaciones de Würch de 1974 (Esparza Ros, 1993) para la MR, la de Von Döbeln modificada por Rocha de 1975 (Esparza Ros) para la MO y las ecuaciones Brozek y Keys de 1951 y Siri de 1956 (Norton \& Olds, 2000) para la MG. La estimación de la densidad corporal se hizo a través de la ecuación de Wilmore y Behnke de 1969 (Norton \& Olds) adecuada para nuestros jugadores según sexo y rango de edad.

A la hora de evaluar a los sujetos se tuvo en cuenta la declaración de Helsinki y los tres principios éticos básicos en investigación humana (respecto, beneficencia y justicia). Se midieron un total de veinte variables incluyendo: edad, peso corporal (en kg), estatura, estatura sentada, envergadura (todas en $\mathrm{cm}$ ), siete pliegues cutáneos (en $\mathrm{mm}$ ), subescapular, tríceps, supraespinal, supracrestal, abdominal, anterior del muslo y medial de la pierna. Dos diámetros (en $\mathrm{cm}$ ), biestiloideo y femoral, y seis perímetros (en $\mathrm{cm}$ ), brazo relajado, antebrazo, torácico mesoesternal, muslo a un $\mathrm{cm}$ del pliegue glúteo, muslo medio y pierna máximo.

El peso corporal fue registrado con una báscula SECA700, exactitud 50 gr. La estatura en bidepestación y sentada se evalúo con estadiómetro portátil Stanley, precisión de $1 \mathrm{~mm}$. La envergadura con cinta métrica Lufkin, precisión de $1 \mathrm{~mm}$. Los perímetros con cinta antropométrica Sanny Medical, precisión de $1 \mathrm{~mm}$. Los diámetros grandes con antropómetro WCS ramas curvas, apertura $50 \mathrm{~cm}$, precisión $1 \mathrm{~mm}$. Los diámetros pequeños con paquímetro corto Campbell 10, apertura $19 \mathrm{~cm}$, precisión $1 \mathrm{~mm}$. Los pliegues cutáneos con plicómetro Harpenden, precisión $0,5 \mathrm{~mm}$.

Las medidas obtenidas fueron volcadas en una base de datos y luego analizadas empleando el paquete estadístico SigmaStat 3.0 para Windows, con registro de la UPO y una aplicación demostrativa del paquete estadístico MedCalc ${ }^{\circledR}$ 8.2.1.0 - 2006.

Para aquellas medidas en escala ordinal, se computaron los siguientes estadísticos: media aritmética, desviación típica, varianza, mediana, moda, valor mínimo y máximo. Como prueba de normalidad, se utilizó la prueba Shapiro-Wilk para muestras dependientes. Se empleo la prueba T o Wilcoxon para contraste de medias con muestras relacionadas. Y para comparaciones múltiples ANOVA o Kruskal-Wallis con el test de Tukey. El nivel de significación establecido fue de 0,5. Finalmente se utiliza el test de Bland \& Altman para determinar la concordancia entre dos métodos. 


\section{RESULTADOS}

La Tabla I muestra la media y desviación típica para las variables antropométricas de los jugadores de bádminton. Los resultados obtenidos en la aplicación de las ecuaciones antropométricas ofrecen datos similares, sin embargo, cuando se comparan estos datos, asociados a la fórmula aplica$\mathrm{da}$, se evidencia un agrupamiento de dos entre las anteriores ecuaciones, evidenciándose diferencias estadísticamente significativas. Este hecho nos lleva a pensar que en el caso de Heymsfield et al. y Poortmans et al. se infraestima la MM, y en las aplicaciones de Martin et al. y Doupe et al. la MM se sobrestima, tal y como se demuestra en la Tabla II y Figura 1 .

Tabla I. Edad y variables antropométricas. Jugadores de bádminton $(\mathrm{n}=37)$. Peso $(\mathrm{kg})$, pliegues cutáneos $(\mathrm{mm})$, resto de variables (cm).

\begin{tabular}{lc}
\hline \multicolumn{1}{c}{ Variable } & media \pm desviación típica \\
\hline Edad & $17,21 \pm 0,94$ \\
Peso & $63,86 \pm 7,07$ \\
Estatura & $169,68 \pm 7,68$ \\
Estatura sentada & $91,56 \pm 3,26$ \\
Envergadura & $174,98 \pm 9,29$ \\
$\Sigma$ de 6 pliegues* & $72,61 \pm 3,77$ \\
Per. Brazo Relajado & $27,06 \pm 1,93$ \\
Per. Antebrazo & $25,55 \pm 1,70$ \\
Per. Tórax & $88,66 \pm 4,72$ \\
Per. Muslo 1 cm & $56,26 \pm 3,77$ \\
Per. Muslo medio & $52,25 \pm 3,02$ \\
Per. Pantorrilla máxima & $36,00 \pm 1,90$ \\
Diam. Biestiloideo & $5,50 \pm 0,36$ \\
Diam. Femoral & $9,41 \pm 0,84$ \\
\hline
\end{tabular}

* 6 pliegues "olímpicos" tricipital, subescapular, abdominal, supraespinal, muslo anterior, medial de la pierna.
Masa muscular en Jugadores de Bádminton

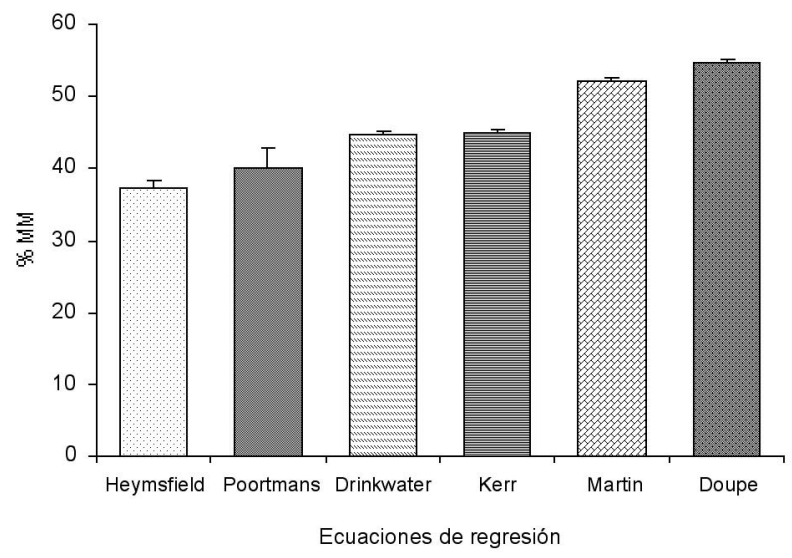

Fig. 1. \% para la MM según seis fórmulas diferentes.

Mediante la aplicación de las dos ecuaciones de regresión para la MG hemos registrado un 13\% en los jugadores estudiados sin obtener diferencias significativas, tal y como se aprecia en los datos aportados en la Tabla III. La MO y MR presentaron un valor de $17 \%$ y $24,10 \%$ respectivamente.

Una vez estimados los cuatro componentes nos encontramos frente a seis modelos fraccionales definidos por las diferentes combinaciones de ecuaciones de regresión. En la Tabla IV se expresan los porcentajes totales de las sumatorias de masas.

A partir del análisis de todas las posibles combinaciones, las seis estrategias dieron valores medios muy cercanos al 100\% del peso real de los deportistas. Si atendemos a las diferencias estadísticas podemos observar que las tácticas que utilizan las ecuaciones de Heymsfield et al. y Poortmas et al. respectivamente, presentan los porcentajes más bajos para la sumatoria de cuatro masas mientras que las tácticas que utilizan las fórmulas de Martin et al. y Doupe et al. reportan valores superiores al $100 \%$.

Tabla II. Masa muscular (MM) expresada en \% del peso total calculado con balanza en jugadores de bádminton según 5 diferentes ecuaciones. $(\mathrm{n}=37)$.

\begin{tabular}{lcccccc}
\hline \multicolumn{1}{c}{ Variable } & $\begin{array}{c}\text { \% MM. } \\
\text { Heymsfiled }\end{array}$ & $\begin{array}{c}\text { \% MM. } \\
\text { Poortmans }\end{array}$ & $\begin{array}{c}\text { \% MM. } \\
\text { Drinkwater }\end{array}$ & \% MM. Kerr & \% MM. Martin & \% MM. Doupe \\
\hline Media & $37,38^{*, \dagger}$ & $40,07^{*, \dagger}$ & $44,63^{*,+, \neq}$ & $44,89^{*,+, \neq}$ & 52,09 & 54,59 \\
Rango & 18,50 & 10,61 & 14,47 & 17,07 & 14,09 & 10,62 \\
Máximo & 47,23 & 44,70 & 50,89 & 52,67 & 59,81 & 60,54 \\
Mínimo & 28,73 & 34,09 & 36,41 & 35,59 & 45,72 & 3,51 \\
Desv. típica & 5,19 & 2,80 & 3,32 & 3,77 & 0,57 & 2,73 \\
Error típico & 0,85 & 0,46 & 0,54 & 0,62 & 0,44 \\
\hline
\end{tabular}

$*$ Diferencias significativas en relación a Doupe, $\mathrm{p}=<0,001$. $\dagger$ Diferencias significativas en relación a Martin, $\mathrm{p}=<0,001$. $\ddagger$ Diferencias significativas en relación a Heymsfield, $\mathrm{p}=<0,001$. 
Tabla III. Masa ósea, residual, y grasa expresados en \% del peso real calculado con balanza. Jugadores badminton $(n=37)$.

\begin{tabular}{cccc}
\hline \multirow{2}{*}{ \% M. Ósea } & \% M. & \multicolumn{2}{c}{$\%$ M. Grasa } \\
\cline { 3 - 4 } & Residual & BROZEK & SIRI \\
\hline $17 \pm 1,93$ & $24,10 \pm 0$ & $13,27 \pm 2,2$ & $13,01 \pm 2,4$ \\
\hline
\end{tabular}

No existen diferencias significativas entre los \% de MG. $\mathrm{p}=<0,005$.

Tabla IV. Porcentaje total de la sumatoria de los componentes estimados en el fraccionamiento corporal en cuatro componentes. Según distintas combinaciones de fórmulas.

\begin{tabular}{lcc}
\hline \multirow{2}{*}{$\begin{array}{c}\text { Masa } \\
\text { Muscular }\end{array}$} & \multicolumn{2}{c}{ Masa ósea + Masa residual : 41,10\% } \\
\cline { 2 - 3 } & Masa grasa (Brozek) & Masa grasa (Siri) \\
\hline Heymsfield & $91,77 \pm 4,86^{*, \dagger}$ & $91,52 \pm 4,86^{*, \dagger}$ \\
Poortmans & $94,46 \pm 2,65^{*, \dagger}$ & $94,21 \pm 2,59^{*, \dagger}$ \\
Drinkwater & $99,02 \pm 3,26^{*, \dagger, \neq}$ & $98,77 \pm 3,18^{*,+, \neq}$ \\
Kerr & $99,29 \pm 3,45^{*, \dagger, \neq}$ & $99,03 \pm 3,45^{*, \dagger, \neq}$ \\
Martin & $106,5 \pm 6,48$ & $106,3 \pm 6,23$ \\
Doupe & $108,9 \pm 2,57$ & $108,7 \pm 2,55$ \\
\hline
\end{tabular}

* Diferencias significativas en relación a Doupe, $\mathrm{p}=<0,001$. $\dagger$ Diferencias significativas en relación a Martin, $p=<0,001$. $\ddagger$ Diferencias significativas en relación a Heymsfield, $\mathrm{p}=<0,001$.

El siguiente paso que realizamos para darle mayor validez interna a nuestros resultados fue examinar el \% de error en la estimación del peso en relación al peso calculado con balanza:

$\%$ Error de estimación del peso $=((\mathrm{PE}-\mathrm{PB}) / \mathrm{PB}) * 100$

Donde: $\mathrm{PE}=$ peso estimado por el método

$\mathrm{PB}=$ peso calculado con balanza

Como revelan los resultados de la Tabla V, los modelos para fraccionar la composición corporal en este tipo de deportistas empleando la ecuación de Kerr para la MM, combinada con la ecuación de Brozek para la MG presentan el menor \% de error de estimación, sin embargo no se aprecian diferencias estadísticamente significativas entre los resultados a un nivel de significación del 5\%.

Tabla V. \% Error de estimación del peso corporal en relación al peso real calculado con balanza.

\begin{tabular}{cccc}
\hline \multicolumn{3}{c}{ MO. Rocha + MR. Würch } \\
\hline \multicolumn{2}{c}{ MM. Drinkwater } & \multicolumn{2}{c}{ MM. Kerr } \\
\hline MG. Brozek & MG. Siri & MG. Brozek & MG. Siri \\
\hline$-0,98 \pm 3,26$ & $-1,23 \pm 3.18$ & $-0,71 \pm 3,25$ & $-0,97 \pm 3,35$ \\
\hline
\end{tabular}

Dada la escasa diferencia en el error de estimación del peso corporal observado con las cuatro estrategias decidimos examinar la concordancia entre los métodos para estimar el peso total (PB calculado con balanza vs. PE con fórmulas de predicción). Figs. 2a, 2b, 2c y 2d
Los gráficos de dispersión resultantes de aplicar el método de Bland \& Altman muestran un error sistemático del segundo método $(\mathrm{PE})$ respecto del primero $(\mathrm{PB})$ en un rango de $0,3 \mathrm{~kg}$ hasta $0,7 \mathrm{~kg}$ según la táctica aplicada, demostrándose que la estrategia Rocha-Würch-Kerr-Brozek (Fig. 2c) presenta la media más baja $(0,3 \mathrm{~kg})$ para las diferencias entre métodos en relación a las otras tres combinaciones, con intervalos de confianza del $95 \%$ entre $-3,9$ y 4,5 $\mathrm{kg}$. Se observa además, una cierta homogeneidad de las diferencias a lo largo del eje horizontal en torno a pesos estimados entre 60 y $75 \mathrm{~kg}$. La tendencia general de las diferencias se torna positiva a medida que disminuye el peso corporal de los sujetos evaluados, lo que indica que el método fraccional ofrece valores de PE menores a los pesos calculados con la balanza.

\section{DISCUSIÓN}

Si bien existen algunos precedentes en la comparación de diferentes metodologías para la estimación de las masas corporales, aún no se ha esclarecido por completo la fiabilidad de los diversos métodos ni se ha llegado a un consenso definitivo en la comunidad científica.

En el presente estudio se ha realizado una amplia revisión bibliográfica y como se puede comprobar al repasar la literatura sobre el tema existe un gran número de ecuaciones destinadas a estimar la MM así como también una gran variabilidad en los métodos aplicados, instrumentación, tamaño muestral, biotipo de sujetos evaluados, rango de edad y procedencia. Todas estas características dejarán entrever la discrepancia existente en las investigaciones realizadas en el ámbito de composición corporal.

Son diversos los autores que han estudiado la composición corporal en jugadores de raqueta, como bádminton (Centeno et al., 1999; Pradas et al., 2006; De Hoyo et al., 2007; Bello \& Garcia, 2007; Garrido, 2004), tenis (Torres et al., 2006; Sánchez-Muñoz et al., 2007) o tenis de mesa (Pradas et al., 2007) por mencionar algunos, pero la gran mayoría se han preocupado principalmente en determinar el componente graso y el somatotipo. Son escasos los investigadores que han examinado la MM en este tipo de jugadores, no obstante utilizan para ello la estrategia de fraccionamiento corporal de cuatro componentes de De Rose y Guimaraes, la cual no calcula la masa muscular a través de ecuaciones de regresión, sino que la obtiene restando la sumatoria de los tres componentes estimados: óseo, residual y graso, al peso calculado con balanza, lo que conlleva en nuestra opinión a errores metodológicos. 

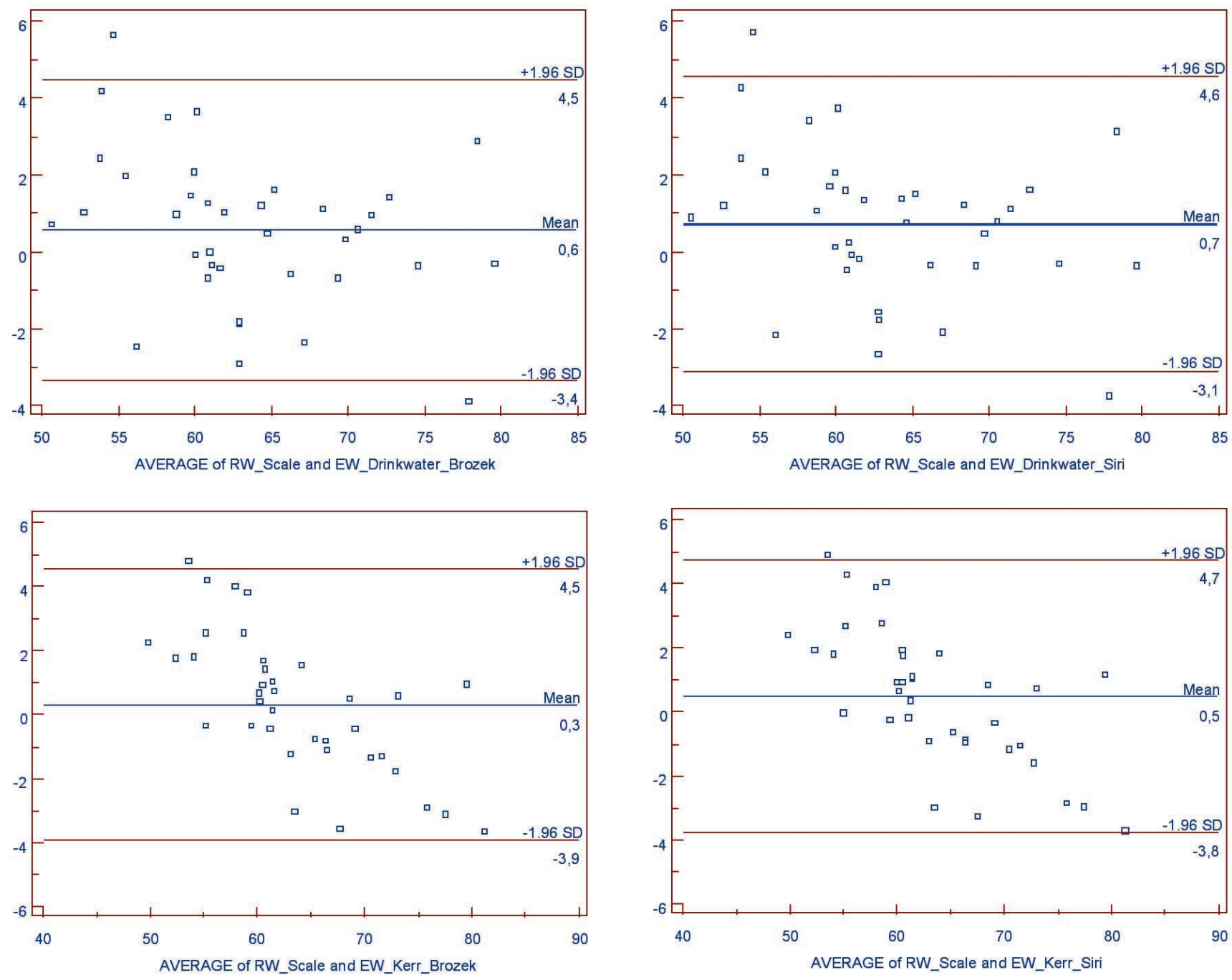

Fig. 2. Concordancia entre métodos para calcular el peso corporal. a) Balanza vs Rocha-Wurch-Drinkwater-Brozek. b) Balanza vs Rocha-Wurch-Drinkwater-Siri. c) Balanza vs Rocha-Wurch-Kerr-Brozek. d) Balanza vs Rocha-Wurch-Kerr-Siri.

Nuestro estudio mostró una menor muscularidad en los jugadores, en relación a los datos aportados por Centeno et al. que examinaron una muestra de 11 jugadores de bádminton de élite de entre 14 y 18 años, con valores 44,7 \pm $3,5 \%$ vs $46,14 \pm 1,68 \%$, respectivamente.

En el campeonato de Andalucía de Selecciones Provinciales Infantiles y Cadete del año 2006, De Hoyo et al., analizaron una muestra de 54 jugadores de bádminton, con los que se realizó un estudio del biotipo y la composición corporal. La MM fue determinada por la estrategia de De Rose y Guimaraes y el valor obtenido fue superior a la de nuestros jugadores, con un $48,01 \%$.

Similares resultados a los observados en los jugadores que controlamos fueron obtenidos en otras dos investigaciones. Una realizada con 47 españoles, jugadores de te- nis de categoría cadetes (14 a 16 años) quienes entrenaban sistemáticamente este deporte durante tres años (Torres $e t$ al.) y otra efectuada en el CeNARD con 69 jugadores argentinos de tenis de alto rendimiento, mostrando un porcentaje para el componente muscular de $44,95 \pm 1,85 \%$ y un $44,9 \%$, respectivamente.

Pradas et al. (2007) recogieron datos antropométricos de 38 jugadores de tenis de mesa infantil de la Real Federación Española y hallaron valores un poco más bajos a los obtenidos en nuestra investigación, obteniendo una MM del $43 \%$.

Varios investigadores han utilizado otras tácticas para la determinación de la composición corporal de jugadores de bádminton, destacando a Garrido que en su trabajo "Composición corporal de jugadores de bádminton" que estudió a 
8 jugadores de nivel nacional (edad 16,68 \pm 7.81 años) y propuso una combinación de ecuaciones para el fraccionamiento tetracompartimental utilizando la fórmula de Faulkner y la de Martin. En este estudio se observó un porcentaje de 40,88 $\pm 5,62 \%$ para la MM.

Bello \& García hallaron en los campeones gallegos de bádminton un valor para la MM del $54,75 \%$, pero no establecieron en su trabajo la fórmula utilizada, por lo que carecemos de documentación que nos sirva para contrastar los resultados.

Sanchez-Muñoz et al. en su trabajo realizado en la Davis Junior Cup evaluaron a 47 jugadores de tenis de 28 equipos nacionales utilizando la ecuación de Poortsman et al. para obtener la masa muscular, encontrando valores similares a Centeno et al. de 46,7 $\pm 1,9 \%$.

En relación con las ecuaciones antropométricas para predecir la MM utilizadas en deportistas de otras modalidades podemos mencionar dos estudios de nivel internacional, el "Kinanthropometry in Aquatic Sports" Carter \& Ackland (1994) realizado en el Campeonato Mundial de Perth'91 y el proyecto "SOKIP'95" (Rienzi et al., 1998) llevado a cabo en el Campeonato Sudamericano de fútbol, así como el estudio realizado por Rodríguez-Bies \& Berral de la Rosa (2006) en gimnastas.

En los dos proyectos internacionales los investigadores utilizaron la misma estrategia para calcular la MM de los deportistas de acuerdo a la ecuación de Martin et al., observándose en los dos estudios elevados porcentajes para el componente muscular similares a los calcula- dos en nuestro estudio con la ecuación de Doupe et al. Para un total de 231 nadadores masculinos obtuvieron un porcentaje de MM de 57,8 $\pm 2,6 \%$. Se evaluaron también 190 jugadores de waterpolo, hallándose en esta muestra una MM de $56 \pm 3,2 \%$. El grupo que presentó la muscularidad más alta fue el de los clavadistas con un valor del 58,6 $\pm 3,6 \%$. Las mediciones realizadas en la Copa América 1995 a 110 futbolistas sudamericanos, pertenecientes a seis selecciones nacionales, permitieron realizar un estudio completo de la morfología de los jugadores, hallándose un valor para el componente muscular del $62,2 \pm 2,9 \%$.

Nuestra propuesta de utilizar la ecuación de Drinkwater o Kerr para la obtención de la masa muscular esquelética en deportistas coincide con la apreciada por Berral et al. (1999) quienes utilizaron una táctica de cuatro componentes para determinar el fraccionamiento de masas corporales en 62 atletas de resistencia de elite. Aplicando diferentes fórmulas para predecir el componente muscular, concluyeron que la estrategia de Drinkwater es la más apropiada para ser utilizada con esa población. El valor que se refleja en este estudio para la MM es de 47,4 $\pm 1.9 \%$.

La aportación fundamental del presente estudio consiste en la comparación múltiple de ecuaciones de regresión para la estimación de la masa muscular y la composición corporal siguiendo una metodología de fraccionamiento en cuatro masas, todo ello a partir de una misma muestra. Con ello nos encontramos en situación óptima para valorar la concordancia entre los distintos resultados y su aplicabilidad en el campo de las ciencias del deporte.

BERRAL DE LA ROSA, F. J.; RODRÍGUEZ-BIES, E. C.; BERRAL DE LA ROSA, C. J.; ROJANO ORTEGA, D. \& LARA PADILLA, E. Comparison of anthropometric equations for estimation muscle mass in badminton player. Int. J. Morphol., 28(3):803-810, 2010.

SUMMARY: The quantification of muscle mass is an area of study that addresses various scientific disciplines from health and sport science as a component of body mass that is in close connection with aspects of health and athletic performance. A variety of methods and equations for their estimation, but implementing them is not always the most appropriate. The purpose of our study is to determine the muscle mass of 37 badminton players of Spain, through anthropometric techniques and finding the most appropriate for this population. We studied 37 badminton players follow the protocol ISAK. Anthropometric variables were evaluated: weight, height, girth, breadth, lengths, heights and skin fold. For the fractionation of body composition in four components were combined different formulas to find the strategy that best fits and matches the estimated weight scale. We used the Wurch's formula for residual mass, Rocha's for bone mass, Brozek's fat mass and to the muscle mass was concluded that the most appropriate for this population with those of Kerr's. Body composition was estimated for our population: $10.8 \%$ bone mass, $15.3 \%$ residual mass, $13.2 \%$ fat mass and $44.9 \%$ muscle mass.

KEY WORDS: Racquet sport; Anthropometry; Body composition. 


\section{REFERENCIAS BIBLIOGRÁFICAS}

Bello, G. \& García, J. L. Perfil Antropométrico y Comparación de los Campeones Gallegos de Bádminton, en Edad Escolar. Comunicación. II Congreso Internacional y XXIV Nacional de Educación Física, 2007.Disponible en:http://www.uibcongres.org/congresos/ ponencia.es.html?cc $=84 \&$ mes $=19 \&$ ordpon $=21$

Berral, F. J.; Viana, B.; Berral, C. J.; Castillo, R.; Salinas, J \& Lancho, J. L. Anthropometric evaluation of muscle mass in athletes. Australia, Fifth IOC World Congress on Sport Sciences, 1999.

Carter, L. \& Ackland, T. R. Kinanthropometry in Aquatic Sports: A Study of World Class Athletes. Champaign, Illinois, Human Kinetics, 1994.

Cattrysse, E.; Zinzen, E.; Caboor, D.; Duquet, W.; Van Roy, P. \& Clarys, J. P. Anthropometric fractionation of body mass: Matiegka revisited. J. Sports Sci., 20(9):717-23, 2002.

Centeno, R.; Naranjo, J. \& Guerra, V. Estudio cineantropométrico del jugador de bádminton de élite juvenil. Arch. Med. Dep., (70):115-9, 1999.

De Hoyo, M.; Sañudo, B.; París, F. \& De la Fuente, L. Estudio del biotipo y la composición corporal en jóvenes jugadores de bádminton. MD, Revista Científica de Medicina del Deporte, 7:9-14, 2007.

Doupe, M. B.; Martin, A. D.; Searle, M. S.; Kriellaars, D. J. \& Giesbrecht, G. G. A new formula for population-based estimation of whole body muscle mass in males. Can.J. Appl. Physiol., 22(6):598-608, 1997.

Drinkwater, D. T. An anatomically derived method for the anthropometric estimation of human body composition. $\mathrm{Ph}$ D. Thesis, Simon Fraser University, 1984.

Esparza Ros, F. Manual de Cineantropometría. Barcelona, Ed. Femede, 1993.

Fernández-Vieitez, J. A.; Álvarez, J. A. \& Willians, L. Áreas musculares del muslo y la pierna estimadas por antropometría y tomografía axial computadorizada en varones adultos. Rev. Cubana Alimen. Nutr., 14(2):10913,2000 .

Garrido, R. Composición corporal de jugadores de bádminton. Revista Digital, 10(68), Buenos Aires, 2004. Disponible en: http://www.efdeportes.com
Heymsfield, S. B.; McManus, C.; Smith, J.; Stevens, V. \& Nixon, D. W. Anthropometric measurement of muscle mass: revised equations for calculating bone-free arm muscle area. Am. J. Clin. Nutr., 36(4):680-90, 1982.

Kerr, D. A. An anthropometric method for fractionation of skin, adipose, bone, muscle and residual tissue masses in males and females age 6 to 77 year. Master Thesis, Simon Fraser University, 1988.

Kim, J.; Shen, W.; Gallagher, D.; Jones, A. Jr.; Wang, Z.; Wang, J.; Heshka, S. \& Heymsfield, S. B. Total-body skeletal muscle mass: estimation by dual-energy X-ray absorptiometry in children and adolescents. Am. J. Clin. Nutr., 84(5):1014-20, 2006.

Lee, R. C.; Wang, Z.; Heo, M.; Ross, R.; Janssen, I. \& Heymsfield, S. B. Total-body skeletal muscle mass: development and cross-validation of anthropometric prediction models. Am. J. Clin. Nutr., 72(3):796-803, 2000.http://www.ncbi.nlm.nih.gov/entrez/utils/ fref.fcgi? PrId=3051\&itool=AbstractPlus def\&uid=10966902\&db=pubmed \&url=http:// w w w . a j c n . o r g / c g i / pmidlookup?view=long \&pmid=10966902

Marfell-Jones, M.; Olds, T.; Stewart, A. \& Carter, L. International standards for anthropometric assessment. Potchefstroom, South Africa, ISAK, 2006.

Martin, A. D.; Spenst, L. F.; Drinkwater, D. T. \& Clarys, J. P. Anthropometric estimation of muscle mass in men. Med. Sci. Sports Exerc., 22(5):729-33, 1990.

Norton, K. \& Olds, T. Estimación antropométrica de la grasa o adiposidad. In: Antropométrica. Buenos Aires, Biosystem, Servicio Educativo, 2000.

Poortmans, J. R.; Boisseau, N.; Moraine, J. J.; Moreno-Reyes, R. \& Goldman, S. Estimation of total-body skeletal muscle mass in children and adolescents. Med. Sci. Sports Exerc., 37(2):316-22, 2005.

Pradas, F.; Martínez, E.; Carrasco, L. \& Herrero, R. Perfil antropométrico, somatotipo, composición corporal y dinamometría manual en jóvenes jugadores de alto nivel de tenis, bádminton y tenis de mesa. IV World Congress Science and Racket Sport. CCD, Revista de Ciencias de la Actividad Física y del Deporte de la Universidad Católica San Antonio, 2(4):73, 2006. 
Pradas F, Carrasco L, Martínez E \& Herrero R. Perfil antropométrico, somatotipo y composición corporal de jóvenes jugadores de tenis de mesa. Rev. Int. Cienc. Deporte, 7(3):11-23, 2007.

Sánchez-Muñoz, C.; Sanz, D. \& Zabala, M. Anthropometric characteristics, body composition and somatotype of elite junior tennis players. Br. J. Sports Med., 41(11):793-9, 2007.

Rienzi, E.; Mazza, J. C.; Carter, J. E. L. \& Reilly, T. SOKIP, Soccer Kinanthropometric Project I. Futbolista sudamericano de élite: Morfología, análisis del juego y performance. Rosario, Editorial Biosystem Servicio Educativo, 1998.

Rodríguez-Bies, E. \& Berral de la Rosa, F. J. Estudio morfológico en gimnastas argentinos de alto rendimiento. Rev. Bras. Cineantropom. Desempenho Hum., 8 (4):16-24, 2006.

Sanada, K.; Kearns, C. F.; Midorikawa, T. \& Abe, T. Prediction and validation of total and regional skeletal muscle mass by ultrasound in Japanese adults. Eur. J. Appl. Physiol., 96(1):24-31, 2006.

Shen, W.; Punyanitya, M.; Wang, Z.; Gallagher, D.; St-Onge, M. P.; Albu, J.; Heymsfield, S. B. \& Heshka, S. Total body skeletal muscle and adipose tissue volumes: estimation from a single abdominal cross-sectional image. J. Appl. Physiol., 97(6):2333-8, 2004.

Tanimoto, Y.; Watanabe, M.; Higuchi, Y.; Hirota, C. \& Kono, K.. Evaluation of the best indicator of muscle mass in community-dwelling elderly persons. Nippon Ronen Igakkai Zasshi., 45(2):213-9, 2008.

Torres, G.; Alacid, F.; Ferragut, C. \& Villaverde, C. Estudio cineantropométrico del jugador de tenis adolescente. CCD, Revista de Ciencias de la Actividad Física y del Deporte de la Universidad Católica San Antonio. 2(4)Supl::71, 2006.

Wang, Z.; Heshka, S.; Pietrobelli, A.; Chen, Z.; Silva, A. M.; Sardinha, L. B.; Wang, J.; Gallager, D. \& Heymsfield, S. B. A new total body potassium method to estimate total body skeletal muscle mass in children. J. Nutr., 137(8):1988-91, 2007.
Dirección para correspondencia:

Francisco José Berral de la Rosa

Departamento de Deporte e Informática

Universidad Pablo de Olavide

Carretera de Utrera km 1. 41013

Sevilla

ESPAÑA

Email: fjberde@upo.es

Recibido : 08-03-2010

Aceptado: 16-07-2010 